\title{
Avaliação da qualidade de vida de adolescentes em tratamento de câncer: Uma revisão integrativa
}

\author{
Quality of life assessment among adolescents in cancer treatment: An integrative review \\ Evaluación de la calidad de vida de adolescentes en el tratamiento de cáncer: Una revisión \\ integradora
}

Recebido: 20/06/2021 | Revisado: 29/06/2021 | Aceito: 03/07/2021 | Publicado: 15/07/2021

\author{
Roberta Dantas Breia de Noronha \\ ORCID: https://orcid.org/0000-0003-4495-6680 \\ Instituto Nacional de Câncer, Brasil \\ E-mail: robertadantasbn@yahoo.com.br \\ Maria Aparecida de Luca Nascimento \\ ORCID: https://orcid.org/0000-0003-0558-460X \\ Universidade Federal do Estado do Rio de Janeiro, Brasil \\ E-mail: gemeas@ centroin.com.br \\ Aline Verônica de Oliveira Gomes Melo \\ ORCID: https://orcid.org/0000-0001-8816-068X \\ Instituto de Puericultura e Pediatria Martagão Gesteira, Brasil \\ E-mail: alinevog@yahoo.com.br \\ Sima Esther Ferman \\ ORCID: https://orcid.org/0000-0002-7076-6779 \\ Instituto Nacional de Câncer, Brasil \\ E-mail: sferman@uol.com.br \\ Sandra Alves do Carmo \\ ORCID: https://orcid.org/0000-0001-8413-0053 \\ Universidade Federal do Rio de Janeiro, Brasil \\ E-mail: drinhaalves@yahoo.com.br
}

\begin{abstract}
Resumo
Objetivo: analisar a qualidade de vida dos adolescentes em tratamento de câncer disponíveis na literatura. Método: Trata-se de uma revisão integrativa da literatura realizada através de busca eletrônica em 07 bases de dados/bibliotecas eletrônicas, no idioma inglês; português ou espanhol, com o recorte temporal de 2015 - 2019. Resultados: foram identificadas 1802 publicações e após a aplicação dos critérios, foram selecionados 13 artigos para composição desta revisão. A síntese das informações extraídas dos artigos foi realizada por meio de instrumento produzido pelas autoras e que permitiu construir as categorias apresentadas. Observa-se que os domínios de qualidade de vida afetados variaram de acordo com o tipo de tratamento recebido. Conclusão: os principais domínios afetados negativamente pelo tratamento do câncer são: o funcionamento social e a saúde física, seguidos do funcionamento emocional e escolar. Constatou-se que o tratamento apenas com cirurgia aparenta ser o tratamento associado a maiores escores de qualidade de vida.
\end{abstract}

Palavras-chave: Adolescente; Câncer; Qualidade de vida; Neoplasias; Oncologia; Enfermagem oncológica.

\begin{abstract}
Objective: to analyze the quality of life of adolescents in cancer treatment available in the literature. Método: revisão integrativa realizada através da busca em 07 bases de dados/bibliotecas eletrônicas, no idioma inglês; português ou espanhol, com o recorte temporal de 2015 - 2019. Method: integrative revision carried out through the search in 07 databases/electronic libraries, in the English language; Portuguese or Spanish, with the time cut from 2015 - 2019. Results: 1802 publications were identified which, after applying the criteria, 13 articles were selected for this review. Results: the synthesis of the information extracted from the articles was made by means of a tool produced by the authors and which allowed the construction of the categories presented. It was observed that the quality of life domains affected varied according to the type of treatment received. Conclusion: the main domain negatively affected by cancer treatment are: social functioning and physical health, followed by emotional and school functioning. It was found that treatment only with surgery appears to be the treatment associated with higher quality of life scores.
\end{abstract}

Keywords: Adolescent; Cancer; Quality of life; Neoplasms; Oncology; Oncology nursing.

\section{Resumen}

Objetivo: analizar la calidad de vida de los adolescentes en el tratamiento del cáncer disponible en la literatura. Método: revisión integradora realizada a través de la búsqueda en 07 bases de datos/bibliotecas electrónicas, en el 
idioma inglés; portugués o español, con el recorte temporal de 2015 a 2019. Resultados: Se identificaron 1802 publicaciones que, después de la aplicación de los criterios, se seleccionaron 13 artículos para esta revisión. Resultados: la síntesis de la información extraída de los artículos se realizó mediante una herramienta elaborada por los autores y que permitió la construcción de las categorías presentadas. Se observa que los ámbitos de la calidad de vida afectados variaban según el tipo de tratamiento recibido. Conclusión: los principales dominios afectados negativamente por el tratamiento del cáncer son: el funcionamiento social y la salud física, seguidos por el funcionamiento emocional y escolar. Se constató que el tratamiento sólo con cirugía parece ser el tratamiento asociado con mayores scores de calidad de vida.

Palabras clave: Adolescente; Cáncer; Calidad de vida; Neoplasias; Oncología; Enfermería oncológica.

\section{Introdução}

São diversas as situações que potencializam o sofrer do adolescente com câncer, tais como: a hospitalização constante; a restrição às atividades cotidianas; a terapêutica agressiva; as alterações da autoimagem e o medo da morte, cuja possibilidade passa a ser mais concreta e está intimamente relacionada com a doença (Rezio, 2016).

Estes adolescentes enfrentam muitos desafios que podem exercer um impacto negativo em seu bem-estar psicossocial, uma vez que desencadeia modificações em múltiplos âmbitos da identidade pessoal e social, potencializando os riscos de desenvolvimento de transtornos emocionais e comportamentais, resultando em prejuízo em diversas dimensões da sua qualidade de vida $(\mathrm{QV})$. Estas repercussões causam também impacto negativo sobre o aprendizado, a capacidade cognitiva e as relações psicossociais (Souza, 2019).

A avaliação da QV permite verificar e entender o impacto global da doença oncológica na vida destes adolescentes e seus familiares, bem como subsidiar os profissionais de saúde na avaliação de intervenções, no planejamento de tratamentos individualizados, na criação de estratégias para melhorar a adesão ao tratamento, predizer e reduzir as complicações e diminuir o tempo de internação hospitalar, assim como contribuem para a construção de políticas públicas, visando à melhora da QV, bem como a priorização na alocação de recursos na saúde (Rezio, 2016; Agathão, 2018)

Nesse contexto, o objetivo deste estudo foi analisar a QV dos adolescentes em tratamento de câncer disponíveis na literatura.

\section{Metodologia}

Trata-se de um estudo de revisão integrativa (RI) de literatura no âmbito da oncologia pediátrica sobre a qualidade de vida dos adolescentes em tratamento de câncer.

A RI consiste em uma revisão bibliográfica sistemática que objetiva traçar uma análise sobre o conhecimento já construído em pesquisas anteriores sobre um determinado tema, de forma a possibilitar a síntese de conhecimento (Mendes et al., 2018; Botelho et al., 2011).

Para esta revisão, seguiram-se as seis etapas indicadas por Mendes et al. (2018) e Botelho et al. (2011): 1) identificação do tema e seleção da hipótese ou questão de pesquisa para elaboração da revisão integrativa; 2) estabelecimento de critérios para inclusão e exclusão de estudos/amostragem ou busca na literatura; 3) definição das informações a serem extraídas dos estudos selecionados/ categorização dos estudos; 4) avaliação dos estudos incluídos na revisão integrativa; 5) análise e interpretação dos resultados; e 6) apresentação da revisão/síntese do conhecimento.

As buscas foram realizadas em dezembro de 2019, norteadas pelas questões de pesquisa formuladas a partir da estratégia PICo de acordo com Cardoso et al. (2019): Quais os domínios da QV são afetados na adolescência pelo tratamento de câncer? Quais os tratamentos estão mais associados à pior QV?

Utilizaram-se as seguintes bibliotecas e bases de dados pelo acesso via Comunidade Acadêmica Federada (CAFe), no Portal de Periódicos da Coordenação Nacional de Pessoal de Nível Superior (CAPES), utilizando a identificação do INCA: Base de Dados em Enfermagem (BDENF) via portal da Biblioteca Virtual de Saúde (BVS); Cummulative Index to Nursing 
and Allied Health Literature (CINAHL); Literatura Latino-Americana e do Caribe em Ciências da Saúde (LILACS); Medical Literature Analysis and Retrieval System Online (MEDLINE) via PubMed®; Scientific Eletronic Library Online (SciELO); SCOPUS (Elsevier) e Web of Science - Coleção Principal (Clarivate Analytics).

Os descritores definidos e utilizados nesta busca estão presentes nos Descritores de Ciências da Saúde (DeCS) e MeSH (Medical Subject Headings): "qualidade de vida", "adolescente", "câncer", "neoplasias" e seus equivalentes em inglês, foram conjugados em pares e/ou trios com o operador boleando and e or para realizar combinações entre os descritores. Para cada base de dados/biblioteca foi utilizada uma estratégia de busca. A estratégia de busca encontra-se no Quadro 1.

Quadro 1 - Estratégia de busca eletrônica para encontrar estudos para a revisão integrativa de literatura, Rio de Janeiro, RJ, Brasil, 2019.

\begin{tabular}{|c|c|}
\hline $\begin{array}{c}\text { Base de } \\
\text { dados/bibliotecas }\end{array}$ & Estratégia de busca \\
\hline BDENF & $\begin{array}{l}\text { Câncer AND Qualidade de vida OR Qualidade de vida AND Adolescente } \\
\text { OR Qualidade de vida AND Adolescente AND Câncer }\end{array}$ \\
\hline CINAHL & (“Adolescent" AND "Quality of life" AND "Cancer") \\
\hline LILACS & $\begin{array}{l}\text { Câncer AND Qualidade de vida OR Qualidade de vida AND Adolescente } \\
\text { OR Qualidade de vida AND Adolescente AND Câncer }\end{array}$ \\
\hline MEDLINE/PubMed & $\begin{array}{l}\text { ("neoplasms"[MeSH Terms] OR "neoplasms"[All Fields]) OR } \\
\text { ("cancer"[All Fields]) AND ("adolescent"[MeSH Terms] OR } \\
\text { "adolescent"[All Fields]) AND (“quality of life" "[MeSH Terms] OR } \\
\text { "quality of life" "[ All Fields]) }\end{array}$ \\
\hline SCIELO & $\begin{array}{l}\text { "Qualidade de vida” AND “Adolescente" OR “Qualidade de vida” AND } \\
\text { "Câncer” OR “Adolescente” AND “Câncer” OR (“Adolescent” AND } \\
\text { "Quality of life”) OR (“Adolescent” AND “Cancer”) }\end{array}$ \\
\hline SCOPUS & $\begin{array}{l}\text { ("Quality of Life" AND “Adolescent" AND "Neoplasms") OR ("Quality } \\
\text { of Life" AND “Adolescent" AND “Cancer") }\end{array}$ \\
\hline WEB OF SCIENCE & $\begin{array}{l}\text { (“Quality of Life” AND “Adolescent” AND “Cancer”) OR (“Quality of } \\
\text { Life” AND “Adolescent”) }\end{array}$ \\
\hline
\end{tabular}

Fonte: Autores. Dados da Pesquisa (2019).

Os critérios de inclusão foram: artigos de pesquisa disponibilizados na íntegra eletronicamente, acesso livre, publicados no idioma inglês; português ou espanhol, com o recorte temporal dos últimos cinco anos (2015 - 2019) visando selecionar estudos atuais e desenvolvidos com a temática de QV de adolescentes em tratamento de câncer.

Os critérios de exclusão adotados foram: estudos que avaliam somente a QV de crianças e adolescentes sobreviventes de câncer ou que não estejam em tratamento oncológico; estudos que abordem somente adultos jovens (19 a 29 anos); que sobre a família, cuidadores e/ou profissionais desses adolescentes; os cuidados paliativos, terminalidade ou fim de vida; relatos de experiência e casos clínicos; artigo de opinião / reflexão; anúncios publicitários; editoriais; cartas ao editor; materiais não científicos; carta-resposta; anais de congresso e estudos de revisão (sistemática; narrativa ou integrativa); teses e dissertações e outros estudos que não respondam as questões da pesquisa. 
Por fim, para a seleção dos estudos, foram seguidas as recomendações do Preferred Reporting Items for Systematic Reviews and Meta-Analyses (PRISMA), que contém quatro etapas: identificação; seleção/triagem; elegibilidade e inclusão (Galvão, 2015).

\section{Resultados}

Foram identificados um total de 1802 publicações em todas as bases de dados/bibliotecas utilizadas neste estudo. Após a aplicação dos critérios de inclusão e exclusão, leitura dos títulos e resumos e, leitura dos textos completos na íntegra, foram selecionados 13 artigos para composição dos resultados desta revisão. A Figura 1 exibe os passos seguidos no processo de seleção dos estudos.

Figura 1 - Fluxo do processo de seleção dos estudos para a revisão integrativa de literatura, elaborado com base nas recomendações PRISMA, Rio de Janeiro, RJ, Brasil, 2019.

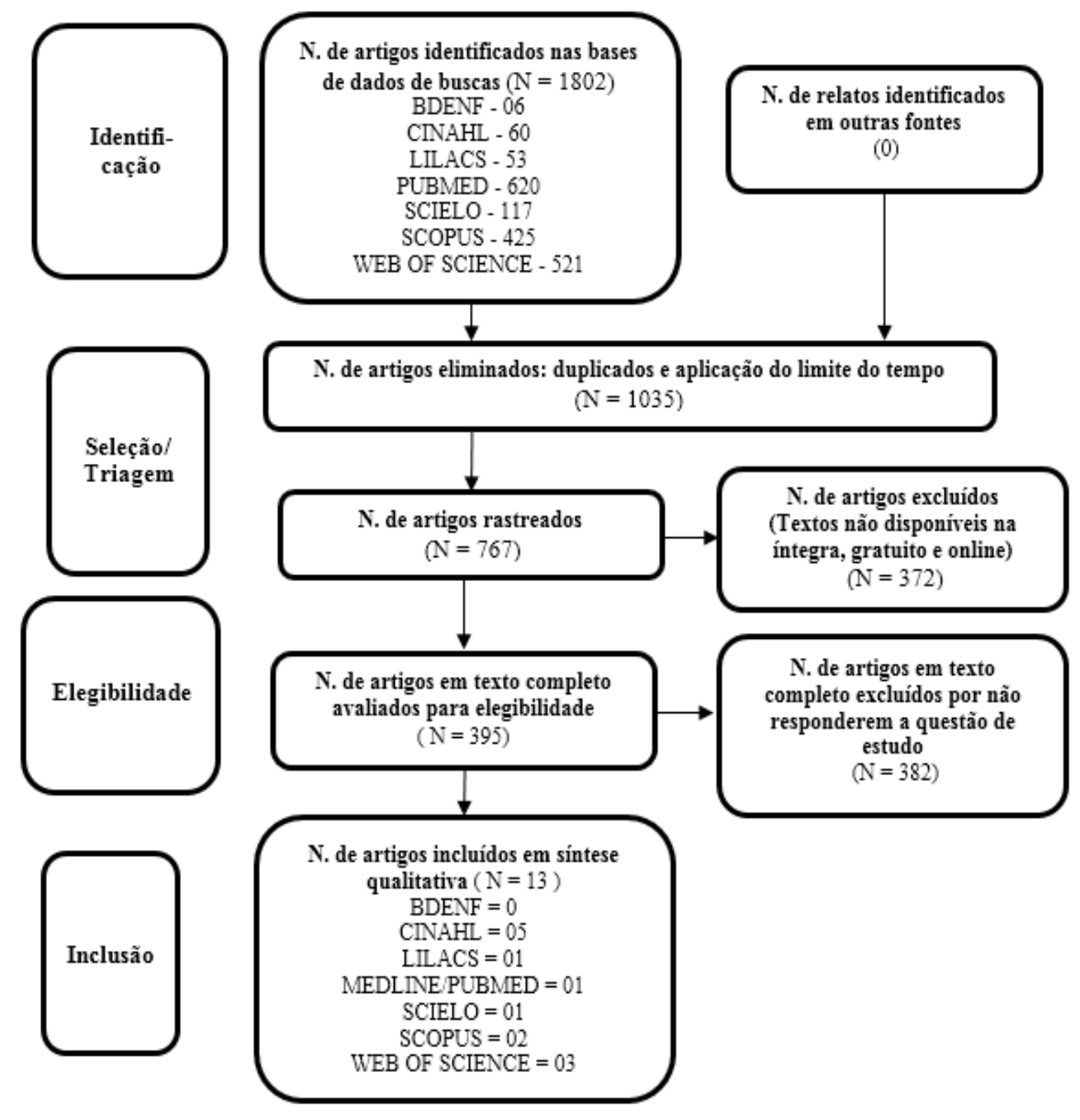

Fonte: Autores. Dados da Pesquisa (2019).

Para análise e interpretação dos dados, realizou-se a síntese das informações extraídas dos artigos incluídos, buscando responder às questões de pesquisa, realizada por meio de instrumento produzido pelas autoras e que permitiu construir as categorias apresentadas. Essa síntese foi organizada em forma de quadros de resultados. 
O Quadro 02 apresenta as principais informações dos artigos incluídos nesta revisão, sendo composto pelos seguintes itens: Número do artigo, Bases de dados; Autores / Revista e Ano / País; Título; Nível de Evidência; Objetivo; Tipo de Estudo; Resultado / Contribuição.

A numeração dada aos artigos (1, 2, 3 ...) será utilizada para identificar os estudos em outros quadros ao longo deste trabalho. Além disso, o nível de evidência foi classificado de acordo com Melnyk (2011): Nível I: revisão sistemática ou metaanálise de todos os relevantes ensaios clínicos randomizados controlados ou provenientes de diretrizes clínicas baseadas em revisões sistemáticas de ensaios clínicos randomizados controlados; Nível II: pelo menos um ensaio clínico randomizado controlado bem delineado; Nível III: ensaios clínicos bem delineados sem randomização; Nível IV: estudos de coorte e de caso-controle bem delineados; Nível V: revisão sistemática de estudos descritivos e qualitativos; Nível VI: um único estudo descritivo ou qualitativo; Nível VII: opinião de autoridades e/ou relatório de comitês de especialistas. Devido à exclusão de estudos de revisão, artigos de níveis de evidências I, V e VII não fizeram parte do processo de seleção. 
Quadro 2 - Principais características dos estudos selecionados.

\begin{tabular}{|c|c|c|c|c|c|c|c|}
\hline $\mathbf{N}^{\circ}$ & $\begin{array}{c}\text { Bases de } \\
\text { Dados }\end{array}$ & $\begin{array}{c}\text { Autores/ Revista/ } \\
\text { Ano/ País }\end{array}$ & Título & Evidência & Objetivo & $\begin{array}{l}\text { Tipo de } \\
\text { estudo }\end{array}$ & Resultado \\
\hline 1 & SCOPUS & $\begin{array}{l}\text { Coça, KL et al / Eur } \\
\text { Eur. J Cancer Care, } \\
2019 \text { / Brasil }\end{array}$ & $\begin{array}{l}\text { Health-related quality of life } \\
\text { of Brazilian children and } \\
\text { adolescents with benign and } \\
\text { malignant solid tumours: A } \\
\text { prospective cohort study } \\
\text { during the first year after } \\
\text { hospital admission. }\end{array}$ & Nível IV & $\begin{array}{l}\text { Avaliar o impacto de tumores sólidos } \\
\text { benignos e malignos e seu tratamento } \\
\text { na QVRS de crianças e adolescentes } \\
\text { acompanhados em um Centro de } \\
\text { Referência em Oncologia Pediátrica no } \\
\text { Rio de Janeiro. }\end{array}$ & $\begin{array}{l}\text { Estudo de } \\
\text { coorte } \\
\text { prospectivo }\end{array}$ & $\begin{array}{l}\text { Crianças e adolescentes com neoplasias } \\
\text { malignas e benignas sofrem mudanças na } \\
\text { qualidade de vida como resultado da } \\
\text { doença e tratamento, mas uma melhoria } \\
\text { foi observado ao longo do tempo. }\end{array}$ \\
\hline 2 & SCOPUS & $\begin{array}{l}\text { Nunes, MDR et al / } \\
\text { Eur J Cancer Care, } \\
2019 \text { / Brasil, EUA e } \\
\text { Portugal. }\end{array}$ & $\begin{array}{l}\text { Pain, sleep patterns and } \\
\text { health-related quality of life } \\
\text { in paediatric patients with } \\
\text { cancer. }\end{array}$ & Nível IV & $\begin{array}{l}\text { Comparar o sono e a QVRS de crianças } \\
\text { e adolescentes com câncer e com dor, } \\
\text { com aquelas que não tem dor durante a } \\
\text { hospitalização. }\end{array}$ & $\begin{array}{l}\text { Estudo de } \\
\text { coorte } \\
\text { prospectivo } \\
\text { e } \\
\text { comparativo }\end{array}$ & $\begin{array}{l}\text { Quase metade dos participantes referiram } \\
\text { dor, contudo, a dor não afetou a qualidade } \\
\text { do sono. Adolescentes do sexo feminino e } \\
\text { pacientes com leucemia/linfoma } \\
\text { apresentaram menor QVRS e duração do } \\
\text { sono. }\end{array}$ \\
\hline 3 & $\begin{array}{l}\text { Web of } \\
\text { Science }\end{array}$ & $\begin{array}{l}\text { Pappot, H et al/ JMIR } \\
\text { Mhealth Uhealth, } \\
2019 \text { / Dinamarca. }\end{array}$ & $\begin{array}{l}\text { Health-Related Quality of } \\
\text { Life Before and After Use of } \\
\text { a Smartphone App for } \\
\text { Adolescents and Young } \\
\text { Adults With Cancer: Pre- } \\
\text { Post Interventional Study. }\end{array}$ & Nível III & $\begin{array}{l}\text { Investigar a viabilidade de um } \\
\text { aplicativo para smartphone entre } \\
\text { adolescentes e adultos jovens (AYA) } \\
\text { com câncer em tratamento ativo e pós- } \\
\text { tratamento, em um teste piloto, } \\
\text { medindo a QVRS antes e após o uso do } \\
\text { aplicativo. }\end{array}$ & $\begin{array}{c}\text { Estudo } \\
\text { Piloto } \\
\text { Pré-pós teste } \\
\text { sem } \\
\text { randomizaçã } \\
\text { o }\end{array}$ & $\begin{array}{l}\text { Mostra a viabilidade e o possível efeito na } \\
\text { QV associado ao uso de uma ferramenta } \\
\text { móvel de saúde em pacientes AYA. As } \\
\text { ferramentas de apoio à saúde móvel são } \\
\text { garantidas para essa população. }\end{array}$ \\
\hline 4 & $\begin{array}{l}\text { Web of } \\
\text { Science }\end{array}$ & $\begin{array}{c}\text { Cox, CL et al / } \\
\text { Pediatr Blood } \\
\text { Cancer, 2018 / EUA. }\end{array}$ & $\begin{array}{l}\text { Modifying bone mineral } \\
\text { density, physical function, } \\
\text { and quality of life in } \\
\text { children with acute } \\
\text { lymphoblastic leukemia. }\end{array}$ & Nível II & $\begin{array}{l}\text { Avaliar a capacidade de uma } \\
\text { fisioterapia e intervenção baseada em } \\
\text { motivação, começando após o } \\
\text { diagnóstico e continuando até o final } \\
\text { do tratamento. }\end{array}$ & $\begin{array}{c}\text { Estudo } \\
\text { Randomizad } \\
\text { o Controlado }\end{array}$ & $\begin{array}{l}\text { A intervenção realizada durante o } \\
\text { tratamento não teve êxito na modificação } \\
\text { da DMO, da função física ou da QVRS. }\end{array}$ \\
\hline 5 & CINAHL & $\begin{array}{l}\text { Samantarath, P et al / } \\
\text { Pacific Rim Int J } \\
\text { Nurs Res, 2018 / } \\
\text { Tailândia. }\end{array}$ & $\begin{array}{l}\text { Multiple Symptoms and } \\
\text { Their Influences on Health- } \\
\text { Related Quality of Life in A } \\
\text { dolescents with Hematologic } \\
\text { Malignancies Undergoing }\end{array}$ & Nível VI & $\begin{array}{l}\text { Descrever as dimensões de ocorrência, } \\
\text { de gravidade e de angústia dos } \\
\text { sintomas desagradáveis, e determinar a } \\
\text { capacidade preditiva desses sintomas } \\
\text { na QVRS em adolescentes tailandeses }\end{array}$ & $\begin{array}{c}\text { Descritivo } \\
\text { Transversal }\end{array}$ & $\begin{array}{l}\text { Os participantes experimentaram vários } \\
\text { sintomas. Os mais prevalentes foram dor, } \\
\text { náuseas / vômitos, falta de apetite, aflição e } \\
\text { perda de cabelo. A tristeza, a perda de } \\
\text { cabelo e a dificuldade em dormir }\end{array}$ \\
\hline
\end{tabular}




\begin{tabular}{|c|c|c|c|c|c|c|c|}
\hline & & & Chemotherapy. & & $\begin{array}{l}\text { com doença hematológica em } \\
\text { tratamento. }\end{array}$ & & $\begin{array}{l}\text { influenciou significativamente na QVRS de } \\
33,2 \% \text {. Os resultados suportam a evidência } \\
\text { de múltiplos sintomas simultâneos } \\
\text { interferindo na QVRS. }\end{array}$ \\
\hline 6 & LILACS & $\begin{array}{l}\text { Anthony, SJ et al / } \\
\text { Qual Life Res, } 2017 \text { / } \\
\text { Canadá. }\end{array}$ & $\begin{array}{l}\text { Quality of life of pediatric } \\
\text { oncology patients: Do } \\
\text { patient-reported outcome } \\
\text { instruments measure what } \\
\text { matters to patients? }\end{array}$ & Nível VI & $\begin{array}{l}\text { Realizar um estudo qualitativo para } \\
\text { fundamentar a teoria da QV e } \\
\text { determinar a adequação de } \\
\text { instrumentos relatados pelo paciente } \\
\text { (PRO) para avaliar a QV em pacientes } \\
\text { pediátricos com câncer e sobreviventes. }\end{array}$ & Descritivo & $\begin{array}{l}\text { Constataram-se problemas importantes } \\
\text { com a validade de conteúdo das escalas } \\
\text { PRO existentes, indicando que as } \\
\text { ferramentas que temos até hoje têm } \\
\text { utilidade limitada na pesquisa e prática } \\
\text { clínica. O desenvolvimento de um novo } \\
\text { instrumento PRO deve ser considerado } \\
\text { para garantir que a validade do conteúdo } \\
\text { seja alcançada. }\end{array}$ \\
\hline 7 & CINAHL & $\begin{array}{l}\text { Nunes, MDR et al/ } \\
\text { European Journal of } \\
\text { Oncology Nursing, } \\
2017 \text { / } \\
\text { Brasil, EUA e } \\
\text { Canadá. }\end{array}$ & $\begin{array}{l}\text { Fatigue and Health related } \\
\text { Quality of life in Children } \\
\text { and Adolescents with } \\
\text { Cancer. }\end{array}$ & Nível IV & $\begin{array}{l}\text { Examinar as diferentes dimensões da } \\
\text { fadiga (geral, sono / descanso, } \\
\text { cognitivo), relacionada com a QVRS } \\
\text { (físico, emocional, cognitivo, social) e } \\
\text { as relações entre fadiga e QVRS em } \\
\text { crianças e adolescentes hospitalizados } \\
\text { com câncer no Brasil. }\end{array}$ & $\begin{array}{c}\text { Corte } \\
\text { Transversal }\end{array}$ & $\begin{array}{l}\text { Crianças e adolescentes com câncer } \\
\text { apresentaram problemas de fadiga } \\
\text { associados com baixa QVRS. Estudos } \\
\text { futuros são recomendados para examinar } \\
\text { intervenções (exercício, lazer, atividades) } \\
\text { que podem aliviar a fadiga e melhorar a } \\
\text { QVRS em pacientes pediátricos com } \\
\text { câncer. }\end{array}$ \\
\hline 8 & $\begin{array}{l}\text { Web of } \\
\text { Science }\end{array}$ & $\begin{array}{l}\text { Rosenberg, AR et al / } \\
\text { Journal of Adolescent } \\
\text { Health, } 2017 \text { / EUA }\end{array}$ & $\begin{array}{l}\text { Intimacy, Substance Use, } \\
\text { and Communication Needs } \\
\text { During Cancer Therapy: A } \\
\text { Report From the "Resilience } \\
\text { in Adolescents and } \\
\text { Young Adults" Study. }\end{array}$ & Nível IV & $\begin{array}{l}\text { Prevalência de intimidade e uso de } \\
\text { substâncias entre adolescentes e } \\
\text { adultos jovens durante a terapia do } \\
\text { câncer. }\end{array}$ & $\begin{array}{l}\text { Coorte, } \\
\text { Prospectivo, } \\
\text { Multicêntric } \\
\quad \text { o }\end{array}$ & $\begin{array}{l}\text { Intimidade sexual e o uso de substância são } \\
\text { comuns entre adolescentes e jovens adultos } \\
\text { com câncer. Os profissionais de saúde } \\
\text { devem normalizar e perguntar sobre estes } \\
\text { comportamentos, a fim de melhor atender } \\
\text { às necessidades do paciente. }\end{array}$ \\
\hline 9 & CINAHL & $\begin{array}{l}\text { Skaczkowski, et al/ } \\
\text { Psycho-Oncology, } \\
2017 \text { / } \\
\text { Austrália. }\end{array}$ & $\begin{array}{l}\text { Do Australian adolescents' } \\
\text { and young adults' } \\
\text { experiences of cancer care } \\
\text { influence their quality of } \\
\text { life? }\end{array}$ & Nível IV & $\begin{array}{l}\text { Examinar a relação entre as } \\
\text { experiências de cuidado no tratamento } \\
\text { dos adolescentes e adultos jovens com } \\
\text { câncer e sua QVRS. }\end{array}$ & $\begin{array}{c}\text { Corte } \\
\text { Transversal }\end{array}$ & $\begin{array}{l}\text { Experiências de cuidados positivos de } \\
\text { atendimento no diagnóstico, durante o } \\
\text { tratamento, durante as internações e ao } \\
\text { finalizar o tratamento, associaram-se a } \\
\text { maior bem-estar funcional, emocional e } \\
\text { social. Experiências positivas de }\end{array}$ \\
\hline
\end{tabular}




\begin{tabular}{|c|c|c|c|c|c|c|c|}
\hline & & & & & & & $\begin{array}{l}\text { comunicação e suporte previram melhor } \\
\text { QVRS nos domínios funcional, emocional, } \\
\text { social e físico, indicando a importância da } \\
\text { informação apropriada à idade e do apoio } \\
\text { emocional no cuidado aos adolescentes e } \\
\text { adultos jovens com câncer. }\end{array}$ \\
\hline 10 & CINAHL & $\begin{array}{c}\text { Ander, M et al / } \\
\text { Psico-Oncology, } \\
2016 / \\
\text { Suécia. }\end{array}$ & $\begin{array}{l}\text { Development of health- } \\
\text { related quality of life and } \\
\text { symptoms of anxiety and } \\
\text { depression among persons } \\
\text { diagnosed with cancer } \\
\text { during adolescence: a 10- } \\
\text { year follow-up study. }\end{array}$ & Nível IV & $\begin{array}{l}\text { Investigar o desenvolvimento da } \\
\text { QVRS e sintomas de ansiedade e } \\
\text { depressão na coorte logo após o } \\
\text { diagnóstico e } \\
\text { até } 10 \text { anos após o diagnóstico. }\end{array}$ & $\begin{array}{c}\text { Corte } \\
\text { Transversal, } \\
\text { Longitudinal }\end{array}$ & $\begin{array}{l}\text { Desenvolvimento da QVRS e sintomas de } \\
\text { ansiedade e depressão parece não linear } \\
\text { entre as pessoas diagnosticadas com câncer } \\
\text { durante a adolescência. Nos sobreviventes, } \\
\text { um aumento nos sintomas de ansiedade é } \\
\text { mostrado, e aproximadamente um terço dos } \\
\text { participantes relatam possível ansiedade. }\end{array}$ \\
\hline 11 & $\begin{array}{l}\text { MEDLI } \\
\text { NE / } \\
\text { PUBME } \\
\text { D }\end{array}$ & $\begin{array}{l}\text { Valachioti, E et al / } \\
\text { Japanese Journal of } \\
\text { Clinical Oncology, } \\
2016 \text { / Grécia. }\end{array}$ & $\begin{array}{l}\text { Assessment of quality of life } \\
\text { of children and adolescents } \\
\text { with cancer during their } \\
\text { treatment. }\end{array}$ & Nível VI & \begin{tabular}{lcccc|} 
Avaliar a & QV & de crianças & e \\
adolescentes & com & qualquer & tipo & de \\
câncer em & todas & as fases & dos & eu \\
tratamento. & & & &
\end{tabular} & $\begin{array}{c}\text { Descritivo } \\
\text { Correlaciona } \\
1\end{array}$ & $\begin{array}{l}\text { Crianças e adolescentes com qualquer tipo } \\
\text { de câncer têm melhores escores de QV no } \\
\text { final do seu tratamento e quando são } \\
\text { apoiados por sua família. }\end{array}$ \\
\hline 12 & SCIELO & $\begin{array}{l}\text { Batalha, LMC; } \\
\text { Fernandes,AM; } \\
\text { Campos, C. / } \\
\text { Esc Anna Nery, } 2015 \\
\text { /Brasil. }\end{array}$ & $\begin{array}{l}\text { Qualidade de } \text { vida em } \\
\text { crianças com } \\
\text { concordância entre crianças } \\
\text { e pais. }\end{array}$ & Nível VI & $\begin{array}{l}\text { Descrever a QVRS de crianças com } \\
\text { câncer (auto e heterorrelato dos pais); } \\
\text { Avaliar a concordância entre auto e } \\
\text { heterorrelato; } \\
\text { Identificar fatores influenciadores das } \\
\text { divergências no auto e heterorrelato da } \\
\text { QVRS da criança }\end{array}$ & $\begin{array}{c}\text { Descritivo } \\
\text { Transversal }\end{array}$ & $\begin{array}{l}\text { A concordância entre auto e heterorrelato } \\
\text { foi moderada, sendo a associação forte. } \\
\text { Variáveis relacionadas com a idade da } \\
\text { criança, sexo, tipo de tumor, tempo de } \\
\text { diagnóstico e número de internações não } \\
\text { influenciaram as divergências entre o auto } \\
\text { e heterorrelato. Uma intervenção com mais } \\
\text { apoio deve ser realizada na resolução das } \\
\text { preocupações, gestão da dor e desconforto } \\
\text { da criança e redução da ansiedade dos pais. } \\
\text { É necessário esclarecer a influência dos } \\
\text { fatores que influenciam as divergências } \\
\text { entre o auto e heterorrelato. }\end{array}$ \\
\hline 13 & CINAHL & $\begin{array}{l}\mathrm{Wu}, \mathrm{W} \text { et al / } \\
\text { Journal of Pediatric } \\
\text { Oncology Nursing, }\end{array}$ & $\begin{array}{l}\text { The Mediating Role of } \\
\text { Resilience on } \\
\text { Quality of Life and Cancer }\end{array}$ & Nível VI & $\begin{array}{l}\text { Descrever os sintomas de angústia, QV } \\
\text { e resiliência em adolescentes com } \\
\text { câncer e determinar se a resiliência é }\end{array}$ & $\begin{array}{l}\text { Descritivo } \\
\text { Transversal }\end{array}$ & $\begin{array}{l}\text { Os resultados confirmam o papel mediador } \\
\text { da resiliência na relação entre angústia e } \\
\text { QV. O desenvolvimento e a avaliação de }\end{array}$ \\
\hline
\end{tabular}


Research, Society and Development, v. 10, n. 8, e42310817392, 2021

(CC BY 4.0) | ISSN 2525-3409 | DOI: http://dx.doi.org/10.33448/rsd-v10i8.17392

\begin{tabular}{|c|c|c|c|c|c|}
\hline & & $\begin{array}{l}2015 \text { / } \\
\text { Taiwan e EUA. }\end{array}$ & $\begin{array}{l}\text { Symptom } \\
\text { Distress in Adolescent } \\
\text { Patients With Cancer. }\end{array}$ & uma variável mediadora na QV. & $\begin{array}{l}\text { intervenções focadas em melhorar a } \\
\text { capacidade de resistência pode ser prático } \\
\text { para enfermeiros. }\end{array}$ \\
\hline
\end{tabular}

Fonte: Autores. Dados da Pesquisa (2019). 
Observa-se que a maior parte, 38,5\% ( $\mathrm{N}=05)$, dos artigos incluídos foram localizados na base de dados CINAHL, seguida da Web of Science com 23,1\% ( $\mathrm{N}=03)$ e da SCOPUS com 15,4\% ( $\mathrm{N}=2)$ do total de artigos. As bases de dados LILACS, Medline/Pubmed e Scielo representam 7,7\% ( $\mathrm{N}=1)$ cada. Não foi incluído nenhum estudo da BDENF. Em relação ao período de publicação selecionado nesta revisão (2015 - 2019), podemos perceber um aumento do número de artigos publicados no ano de 2017 com 30,8\% ( $=4)$ e 2019 com 23,1\% ( N = 3), seguidos dos anos de 2015,2016 e 2018 com 15,4\% cada $(\mathrm{N}=2)$. No que diz respeito ao idioma das publicações, 12 estavam redigidas em inglês $(92,3 \%)$, uma em português $(7,7 \%)$ e nenhuma em espanhol. Embora o idioma inglês tenha predominado, as publicações foram realizadas com amostras de diversos países.

No que diz respeito aos periódicos, os artigos foram publicados em 11 periódicos diferentes e, dentre esses, apenas dois (European Journal of Cancer Care e Psico-Oncology) apresentam duas publicações relacionadas a temática desta revisão. Por fim, no que tange o nível de evidência dos estudos incluídos, observa-se que 46,2\% dos estudos eram de nível IV (N = 06), 38,5\% ( $\mathrm{N}=05)$ eram de nível VI. Apenas um estudo era de nível II e outro de nível III, representando 7,7\% cada.

O Quadro 03 apresenta as principais informações relacionadas aos participantes de cada um dos estudos selecionados, como o número de participantes, sexo, idade, principais tipos de câncer dos participantes, tratamento recebido para tratar o câncer e domínios da QV afetados pelo tratamento do câncer.

Quadro 3 - Principais características dos participantes dos estudos selecionados.

\begin{tabular}{|c|c|c|c|c|c|c|}
\hline $\mathbf{N}^{\circ}$ & Participantes & Sexo & $\begin{array}{l}\text { Faixa } \\
\text { Etária }\end{array}$ & Tipo de Câncer & Tratamento Recebido & $\begin{array}{c}\text { Domínios de QV } \\
\text { Afetados }\end{array}$ \\
\hline 1 & 132 & $\begin{array}{l}59 \mathrm{M} \\
73 \mathrm{~F}\end{array}$ & $2-17$ & $\begin{array}{l}\text { Tumores no: SNS; } \\
\text { Ossos e Outros } \\
\text { tumores sólidos }\end{array}$ & $\begin{array}{l}\text { Cirurgia; Cirurgia }+ \\
\text { quimioterapia; Cirurgia } \\
+\quad \text { radioterapia }+ \\
\text { quimioterapia }\end{array}$ & $\begin{array}{l}\text { QVRS - Saúde física, } \\
\text { Funcionamento } \\
\text { emocional, social e } \\
\text { escolar }\end{array}$ \\
\hline 2 & 118 & $\begin{array}{l}40 \mathrm{M} \\
78 \mathrm{~F}\end{array}$ & $8-18$ & $\begin{array}{l}\text { Leucemia } \\
\text { linfoma; Sarcomas; } \\
\text { Outros }\end{array}$ & Quimioterapia & $\begin{array}{l}\text { QVRS - Saúde física, } \\
\text { Funcionamento } \\
\text { emocional, social e } \\
\text { escolar }\end{array}$ \\
\hline 3 & 20 & $\begin{array}{l}6 \mathrm{M} \\
14 \mathrm{~F}\end{array}$ & $15-29$ & $\begin{array}{l}\text { Linfoma; Mama; } \\
\text { Cabeça e pescoço; } \\
\text { Outros }\end{array}$ & NR & $\begin{array}{l}\text { QVRS - Saúde física, } \\
\text { Funcionamento } \\
\text { emocional, social e } \\
\text { escolar }\end{array}$ \\
\hline 4 & 73 & $\begin{array}{l}34 \mathrm{M} \\
19 \mathrm{~F}\end{array}$ & $4-19$ & $\begin{array}{l}\text { Leucemia linfoide } \\
\text { aguda }\end{array}$ & $\begin{array}{l}\text { Fisioterapia e } \\
\text { intervenção } \\
\text { motivacional }\end{array}$ & $\begin{array}{l}\text { QVRS - Saúde física e } \\
\text { mental, funcionamento } \\
\text { cognitivo e social }\end{array}$ \\
\hline 5 & 94 & & $10-18$ & Leucemia e linfoma & Quimioterapia & $\begin{array}{l}\text { QVRS - Saúde física, } \\
\text { Funcionamento } \\
\text { emocional, social e } \\
\text { escolar }\end{array}$ \\
\hline 6 & 37 & $\begin{array}{l}18 \mathrm{M} \\
19 \mathrm{~F}\end{array}$ & $8-18$ & $\begin{array}{c}\text { Leucemia e } \\
\text { linfoma; Outros }\end{array}$ & Todos & $\begin{array}{l}\text { QV - Saúde física, } \\
\text { psicológica e social }\end{array}$ \\
\hline
\end{tabular}




\begin{tabular}{|c|c|c|c|c|c|c|}
\hline & & & & tumores sólidos & & \\
\hline 7 & 38 & $\begin{array}{r}25 \mathrm{M} \\
13 \mathrm{~F}\end{array}$ & $8-18$ & $\begin{array}{c}\text { Leucemia e } \\
\text { linfoma; Sarcomas; } \\
\text { Tumor no SNS; } \\
\text { Outros }\end{array}$ & Quimioterapia & $\begin{array}{l}\text { QVRS - Saúde física, } \\
\text { Funcionamento } \\
\text { emocional, social e } \\
\text { escolar }\end{array}$ \\
\hline 8 & 35 & $\begin{array}{c}20 \mathrm{M} \\
15 \mathrm{~F}\end{array}$ & $14-25$ & $\begin{array}{l}\text { Leucemia linfoide } \\
\text { aguda; Linfoma de } \\
\text { Hodgkin; Sarcomas; }\end{array}$ & Quimioterapia & $\begin{array}{l}\text { QV - Funcionamento } \\
\text { social e psicológico }\end{array}$ \\
\hline 9 & 207 & $\begin{array}{l}75 \mathrm{M} \\
132 \mathrm{~F}\end{array}$ & $15-24$ & $\begin{array}{c}\text { Leucemia e } \\
\text { linfoma; Outros }\end{array}$ & $\begin{array}{c}\text { Cirurgia; Quimioterapia; } \\
\text { Radioterapia }\end{array}$ & $\begin{array}{l}\text { QV - Saúde física, } \\
\text { psicológica e social }\end{array}$ \\
\hline 10 & 61 & $\begin{array}{l}37 \mathrm{M} \\
24 \mathrm{~F}\end{array}$ & 13-19 & $\begin{array}{c}\text { Leucemia e } \\
\text { linfoma; Sarcomas; } \\
\text { Tumor no SNS; } \\
\text { Outros }\end{array}$ & Quimioterapia & $\begin{array}{l}\text { QVRS - Saúde física e } \\
\text { mental, Funcionamento } \\
\text { emocional, social e } \\
\text { escolar }\end{array}$ \\
\hline 11 & 56 & $\begin{array}{l}32 \mathrm{M} \\
24 F\end{array}$ & $8-20$ & $\begin{array}{c}\text { Leucemia e } \\
\text { linfoma; Tumores } \\
\text { sólidos }\end{array}$ & $\mathrm{NR}$ & $\begin{array}{c}\text { QV - Saúde física, } \\
\text { funcionamento } \\
\text { cognitivo, psicológico, } \\
\text { social e familiar }\end{array}$ \\
\hline 12 & 75 & $\begin{array}{l}54 \mathrm{M} \\
21 F\end{array}$ & $8-17$ & NR & NR & $\begin{array}{c}\text { QVRS - Saúde física e } \\
\text { mental, funcionamento } \\
\text { cogntivo e social }\end{array}$ \\
\hline 13 & 40 & $\begin{array}{r}25 \mathrm{M} \\
15 F\end{array}$ & $13-20$ & $\begin{array}{c}\text { Leucemia e } \\
\text { linfoma; Sarcoma }\end{array}$ & NR & $\begin{array}{l}\text { QV - Saúde física, } \\
\text { funcionamento } \\
\text { cognitivo, psicológico, } \\
\text { social e familiar }\end{array}$ \\
\hline
\end{tabular}

Nota: SNS - Sistema Nervoso Central; F - Feminino; M - Masculino; NR - Não Reportado. Fonte: Autores. Dados da Pesquisa (2019).

Observa-se que o número de participantes dos estudos variou entre 20 e 207. A soma dos participantes de todos os estudos chega a um $\mathrm{N}=986$. Em relação aos tipos de câncer dos participantes, a leucemia e o linfoma foram os diagnósticos mais frequentes, reportados como um dos principais tipos de câncer em 10 dos estudos incluídos.

No que diz respeito ao tratamento recebido, a quimioterapia foi o tratamento mais utilizado $(\mathrm{N}=07)$, seguida da radioterapia e cirurgia, ambas citadas em dois estudos. Um dos estudos não mencionou quais os tipos de tratamentos utilizados, informando apenas que pacientes com câncer realizando qualquer tipo de tratamento foram incluídos. Outro estudo utilizou apenas fisioterapia e intervenção motivacional. Além disso, quatro dos artigos selecionados não reportaram dados relacionados ao tratamento recebido pelos participantes.

Em relação aos domínios de QV afetados pelo tratamento do câncer, evidenciou-se que o funcionamento social $(\mathrm{N}=$ 13) e a saúde física $(\mathrm{N}=12)$ foram os domínios mais afetados, seguido do funcionamento emocional e escolar $(\mathrm{N}=06)$, saúde/funcionamento psicológico $(\mathrm{N}=05)$, funcionamento cognitivo $(\mathrm{N}=4)$, saúde mental $(\mathrm{N}=03)$, funcionamento familiar $(\mathrm{N}=02)$. Algumas semelhanças podem ser observadas nas nomenclaturas dos domínios de QV reportados pelos estudos incluídos, isto ocorre devido aos diferentes instrumentos utilizados para mensurar a QV. 


\section{Discussão}

Observa-se nos resultados encontrados, que os domínios de QV afetados variaram de acordo com o tipo de tratamento recebido. Com isso, com o intuito de realizar uma discussão relevante, elaboramos categorias baseadas nos tipos de tratamento, nos quais os artigos selecionados foram agrupados por similaridade de conteúdo.

\section{Cirurgia e a qualidade de vida dos adolescentes em tratamento para o câncer}

A cirurgia foi utilizada como tratamento apenas nos estudos de Coça et al. (2019) e Skaczkowski (2018). No entanto, quando somado o número de participantes de ambos os estudos, observa-se que eles representam 33\% ( $\mathrm{N}=325)$ da soma total dos 13 artigos selecionados. Observou-se, nas duas pesquisas, que os pacientes que receberam apenas tratamento cirúrgico apresentaram maiores escores nos domínios de saúde física, funcionamento emocional, social e escolar, e saúde psicológica.

Para os autores citados acima, os escores de QV foram maiores quando comparados aos pacientes que receberam quimioterapia, radioterapia, ou tratamentos combinados (e.g. quimioterapia + cirurgia). Este achado corrobora com Si et al. (2020) que observaram melhora da QV após a realização de intervenções cirúrgicas em pacientes com câncer de mama e em adolescentes com tumores ósseos.

\section{Quimioterapia e a qualidade de vida dos adolescentes em tratamento para o câncer}

A maior parte dos estudos incluídos $(\mathrm{N}=07)$ utilizou a quimioterapia como forma de tratamento do câncer. Coça et al. (2019) e Skaczkowski (2018) compararam a quimioterapia com outras formas de tratamento, sendo observado que os pacientes em tratamento com quimioterapia apresentam os piores escores de QV nos domínios de saúde física, funcionamento emocional, social e escolar quando comparada a qualquer outra forma de tratamento.

Embora os outros cinco estudos (Nunes et al., 2017; 2018; Samantarath et al., 2018; Rosenberg et al., 2016; Ander, 2016) tenham incluído pacientes que realizavam tratamento apenas com quimioterapia, foi possível observar prejuízos significativos nos escores de diversos domínios de QV, principalmente nos domínios de saúde física, funcionamento emocional, social e escolar, sendo os últimos dois, os mais afetados. Tal achado corrobora com os resultados de Fernández et al. (2017) e Saad (2019). No entanto, de acordo com INCA (2020) a quimioterapia é uma das principais e mais eficazes formas de tratamento do câncer, tanto sozinha como combinada com algum outro tipo de tratamento (e.g. cirurgia, radioterapia, transplante de medula óssea).

\section{Outros tratamentos e fatores associados a qualidade de vida dos adolescentes em tratamento para o câncer}

Embora somente os estudos de Skaczkowski (2018) e Coça et al. (2019) tenham reportado dados relacionados a QV de pacientes que receberam formas diferentes de tratamento, observou-se que depois do tratamento apenas com cirurgia, o tratamento combinado com cirurgia, quimioterapia e radioterapia foi o mais associado aos maiores escores de QV. Já no tratamento combinado com cirurgia e quimioterapia ou tratamentos apenas com quimioterapia ou radioterapia, foram observados escores significativamente menores de QV.

Pappot et al. (2019) utilizaram em seu estudo, um app para telefone móvel que objetiva aumentar a QV dos pacientes em tratamento para o câncer. Contudo, concluiu-se que a QV dos pacientes se manteve estável entre o baseline e após 6 semanas de intervenção. Foi observado aumento nos escores de QV apenas no grupo de pacientes que já havia finalizado o tratamento para o câncer. Além disso, os autores não reportaram quais os tratamentos padrões (i.e. quimioterapia, cirurgia, radioterapia) aos quais os participantes foram submetidos. Estes achados foram similares aos estudos de Coça et al. (2019), 
Ander (2016) e Vlachioti (2016) que reportaram que os participantes apresentaram maiores escores de QV somente após o término dos tratamentos.

Ainda sobre os tratamentos, o estudo de Cox et al. (2018) testou a eficácia de uma intervenção com fisioterapia e intervenção motivacional como tentativa de aprimorar a QV de adolescentes com diagnóstico de Leucemia linfoide aguda, contudo, os resultados obtidos demonstraram que a intervenção não foi eficaz. Os autores deste estudo também não informaram qual o tratamento padrão para o tratamento do câncer foi utilizado.

Uma revisão sobre a utilização de tratamentos combinados realizada por Goetze et al. (2018) sugere que o melhor tratamento varia de acordo com cada tipo de câncer, os tratamentos combinados são muito mais eficazes do que apenas o tratamento cirúrgico. Embora isso possa impactar negativamente na QV dos pacientes, Goetze et al. (2018), Al-Batran et al. (2018), Macciò (2012), Ychou et al. (2011) e Songun et al. (2010) destacam que a taxa de sobrevivência apenas com intervenção cirúrgica é de $25 \%$. Logo, os tratamentos combinados seriam recomendados mesmo em detrimento da QV do paciente a curto e longo prazo.

Goetze et al. (2018) e Epstein et al. (2010) relatam que há necessidade de se considerar não apenas os impactos dos tratamentos na QV, mas também a probabilidade de sua eficácia, discutindo sempre seus prós e contras com o paciente e familiares.

Além dos tipos de tratamento foram observados alguns outros fatores que influenciaram os escores de QV. Dois deles foram os sintomas físicos, sendo evidenciado que pacientes com dor no estudo de Nunes et al. (2019), perda de cabelo, dificuldade para dormir no estudo de Samantarath et al. (2018), fadiga no estudo de Nunes et al. (2017) e náusea no estudo de Batalha, Fernandes e Campos (2015) apresentaram menores escores de QV. Destaca-se ainda que quanto maior a duração da quimioterapia maiores foram os escores de fadiga e menores os de QV (Nunes et al., 2017).

Quanto aos sintomas psicológicos, constatou-se nos adolescentes, altos escores de tristeza no estudo de Samantarath et al. (2018), preocupação no estudo de Batalha, Fernandes e Campos (2015), angústia e baixa resiliência no estudo de Wu et al. (2015), apresentando, também, menores escores de QV. Ressalta-se que a esperança foi considerada uma importante estratégia de enfrentamento entre pacientes com câncer nos estudos de Getze et al. (2018) e Li et al. (2018), os autores relatam que um alto nível de esperança está associado com menor nível de ansiedade e depressão, maior suporte social e melhor QV.

Outro ponto importante a ser destacado é a necessidade do desenvolvimento de capacitações voltada para os profissionais de saúde sobre o cuidado com os adolescentes com câncer, segundo Skarczkowski et al. Existe uma correlação positiva entre o cuidado recebido da equipe de saúde durante a internação hospitalar e melhores escores de QV (i.e. saúde física, emocional e social). Vários estudos demonstraram a influência dos profissionais de saúde para a potencialização da esperança de pacientes com câncer, contribuindo para a melhora dos escores de QV (Li et al., 2018). A partir disso, será possível desenvolver intervenções que contribuam com a melhora da QV dos pacientes oncológicos. Li et al. (2018) reforça que as intervenções de enfermagem são estratégias úteis para aumentar a esperança nas pessoas com câncer.

No que tange aos fatores reportados como mediadores da QV, o estudo de Samantarath et al. (2018) menciona os sintomas de perda de cabelo, dificuldade para dormir e sentimento de tristeza. Já o estudo de Vlachioti (2016) cita o suporte familiar; Náuseas, preocupações dos adolescentes e dos pais são citados por Batalha, Fernandes e Campos (2015) e a esperança e a resiliência foram mencionados por Li et al. (2018). Wu Wei-Wen et al. (2015) ressaltam que quanto maior for a resiliência, menores serão os impactos dos sintomas do câncer e de seu tratamento nos adolescentes.

Cabe destacar, que os estudos de Li et al. (2018), Vlachioti (2016), Batalha, Fernandes e Campos (2015), Wu WeiWen et al. (2015), Samantarath et al. (2018) e Rosenberg et al. (2017) reforçam a necessidade de desenvolver estratégias de intervenção que objetivem manejar os sintomas, as preocupações dos pais e sobretudo o aprimoramento da capacidade de resiliência dos adolescentes visando a melhora da qualidade de vida. 


\section{Considerações Finais}

Conclui-se que os principais domínios da QV afetados negativamente pelo tratamento do câncer são: o funcionamento social e a saúde física, seguidos do funcionamento emocional e escolar. Além disso, constatou-se que o tratamento apenas com cirurgia aparenta ser o tratamento associado a maiores escores de QV.

Observa-se carência de estudos nacionais sobre a QV dos adolescentes em tratamento para o câncer, tendo em vista que apenas dois dos estudos incluídos foram realizados com amostra brasileira. No entanto, para além da necessidade de realização de mais estudos que investiguem a QV dos adolescentes com câncer, sugere-se que tais estudos levem em consideração as associações entre QV e tipos de câncer, visto que a literatura aponta que cada tipo de câncer necessitará de um tratamento diferente, o qual pode ser mais ou menos agressivo e por consequência terá um impacto maior ou menor na QV dos pacientes oncológicos.

Além disso, sugere-se a realização de intervenções com a equipe de saúde, com o intuito de aprimorar a comunicação e orientar melhor sobre os tipos de tratamentos disponíveis e seus impactos e o manejo/atenção fornecida aos adolescentes com câncer. Nessa mesma linha, é fundamental a realização de intervenções com os pais/cuidadores dos adolescentes, sobretudo visando a redução dos sintomas de ansiedade, angústia e preocupação. Por fim, destaca-se a necessidade de intervenções que objetivem o aumento da resiliência e enfrentamento dos sintomas decorrentes dos tratamentos propostos aos adolescentes com câncer.

\section{Referências}

Agathão, B. T., Reichenheim, M. E., Moraes, C. L. (2018). Qualidade de vida relacionada à saúde de adolescentes escolares. Ciência \& Saúde Coletiva, 23(2), 659-668. https://doi.org/10.1590/1413-81232018232.27572016

Al-Batran, S. E., Goetze, T. O., Mueller, D. W., et al. (2017). The RENAISSANCE (AIO-FLOT5) trial: effect of chemotherapy alone vs. chemotherapy followed by surgical resection on survival and quality of life in patients with limited-metastatic adenocarcinoma of the stomach or esophagogastric junction - a phase III trial of the German AIO/CAO-V/CAOGI. BMC Cancer, 17(1), 893. 10.1186/s12885-017-3918-9

Ander, M. (2016). Development of health-related quality of life and symptoms of anxiety and depression among persons diagnosed with cancer during adolescence: a 10-year follow-up study. Psycho-Oncology, 25, 582-589. 10.1002/pon.3965

Anthony, S. J. et al. (2017). Quality of life of pediatric oncology patients: Do patient-reported outcome instruments measure what matters to patients? Qual Life Res, 26, 273-281. https://doi.org/10.1007/s11136-016-1393-4

Batalha, L. M. C., Fernandes, A. M., Campos, C. (2015). Qualidade de vida em crianças com câncer: concordância entre crianças e pais. Esc Anna Nery, 19(2), 292-296. 10.5935/1414-8145.20150039

Botelho, L. L. R., Cunha, C. C. A., Macedo, M. (2011). O método da revisão integrativa nos estudos organizacionais. Gest Soc, 5(11), 121-136. https://doi.org/10.21171/ges.v5i11.1220

Cardoso, V., Trevisan, I., Cicolella, D. A., \& Waterkemper, R. (2019). Revisão sistemática de métodos mistos: método de pesquisa para a incorporação de evidências na enfermagem. Texto contexto - enferm, 28, e20170279. https://doi.org/10.1590/1980-265x-tce-2017-0279

Coça, K. L. et al. (2019). Health-related quality of life of Brazilian children and adolescents with benign and malignant solid tumours: A prospective cohort study during the first year after hospital admission. Eur Eur. J Cancer Care, 28(5), e13102. https://doi.org/10.1111/ecc.13102

Cox, C. L. et al. (2018). Modifying bone mineral density, physical function, and quality of life in children with acute lymphoblastic leukemia. Pediatr Blood Cancer, 65(4), e10.1002. https://doi.org/10.1002/pbc.26929.

Epstein, R. M., Duberstein, P. R., Fenton, J. J. et al. (2017). Effect of a patient-centered communication intervention on oncologist-patient communication, quality of life, and health care utilization in advanced cancer: the VOICE randomized clinical trial. JAMA oncology, 3(1), 92-100. 10.1001/jamaoncol.2016.4373

Fernández, K. S., Schwartz, C. L., Chen, L., Constine, L. S., Chauvenet, A. \& de Alarcón, P. A. (2017). Outcome of adolescents and young adults compared to children with Hodgkin lymphoma treated with response-based chemotherapy on pediatric protocols: A Children's Oncology Group report. Pediatric blood \& câncer, 64(12), e26681. 10.1002/pbc.26681

Galvão, T. F., Pansani, T. S. A., \& Harrad, D. (2015). Principais itens para relatar Revisões sistemáticas e meta-análises: a recomendação PRISMA. Epidemiol Serv Saude, 24(2), 335-342. https://doi.org/10.5123/S1679-49742015000200017

Goetze, O. T., Al-Batran, S. E., Chevallay, M. \& Mönig, S. P. (2018). Multimodal treatment in locally advanced gastric cancer. Updates in surgery, 70(2), 173-179. 10.1007/s13304-018-0539-Z 
Research, Society and Development, v. 10, n. 8, e42310817392, 2021

(CC BY 4.0) | ISSN 2525-3409 | DOI: http://dx.doi.org/10.33448/rsd-v10i8.17392

Instituto Nacional de Câncer José Alencar Gomes da Silva. (2020). ABC do câncer: abordagens básicas para o controle do câncer / Instituto Nacional de Câncer José Alencar Gomes da Silva. INCA, 2020. Manual. Disponível em: https://www.inca.gov.br/sites/ufu.sti.inca.local/files//media/document//livro-abc6-edicao-2020.pdf

Li, P., Guo, Y. J., Tang, Q., \& Yang, L. (2018). Effectiveness of nursing intervention for increasing hope in patients with cancer: a metaanalysis. Rev. LatinoAm. Enfermagem, 26, e2937. http://dx.doi.org/10.1590/1518-8345.1920.2937

Macciò, A., Madeddu, C., Gramignano, G., Mulas, C., Floris, C., Sanna, E. et al. (2012). A randomized phase III clinical trial of a combined treatment for cachexia in patients with gynecological cancers: evaluating the impact on metabolic and inflammatory profiles and quality of life. Gynecologic oncology, 124(3), 417-425. 10.1016/j.ygyno.2011.12.435

Melnyk, B. M. \& Fineout-overholt, E. (2011). Evidence-based practice in nursing and health care: a guide to best practice. Lippincott, Williams \& Wilkins.

Mendes, K. D. S., Silveira, R. C. C. P., \& Galvão, C. M. (2008). Revisão integrativa: Método de pesquisa para a incorporação de evidências na saúde e na enfermagem. Texto Contexto Enferm, 17(4), 758-764. https://doi.org/10.1590/S0104-07072008000400018

Nunes, M. D. R. et al. (2019). Pain, sleep patterns and health-related quality of life in paediatric. Eur J Cancer Care, 28(4), e13029. . https://doi.org/10.1111/ecc.13029

Nunes, M. D. R. et al. (2017). Fatigue and health related quality of life in children and adolescents with cancer. Europen Journal of Oncology Nursing, 2017, 29, 39-46. https://doi.org/10.1016/j.ejon.2017.05.001

Pappot, H. et al. (2019). Health-Related Quality of Life Before and After Use of a Smartphone App for Adolescents and Young Adults With Cancer: Pre-Post Interventional Study. JMIR Mhealth Uhealth, 7(10), e13829. 10.2196/13829

Rezio, M. A. (2016). Qualidade de vida relacionada à saúde de adolescentes com doenças crônicas. Dissertação de Mestrado. Faculdade Federal de Goiás, Faculdade de Nutrição (Fanut). Programa de Pós-Graduação em Nutrição e Saúde, Goiás, 2016.

Rosenberg, A. R. et al. (2016). Intimacy, Substance Use, and Communication Needs During Cancer Therapy: A Report From the "Resilience in Adolescents and Young Adults" Study. Journal of Adolescent Health, 60, 93-99. 10.1016/j.jadohealth.2016.08.017

Saad, Al-Nassan et al. (2019). Health-Related Quality of Life Among Jordanian Adolescent Cancer Patients Receiving Active Treatment. Asian Pacific Journal of Cancer Prevention, 20(10), 3107-3111. 10.31557/APJCP.2019.20.10.3107

Samantarath, P. et al. (2018). Multiple Symptoms and Their Influences on Health-Related Quality of Life in Adolescents with Hematologic Malignancies Undergoing Chemotherapy. Pacific Rim Int J Nurs Res, 22(4), 319-331. https://he02.tci-thaijo.org/index.php/PRIJNR/article/view/88211/109033

Si, Y., Yuan, P., Hu, N., Wang, X., Ju, J., Wang, J., Ma, F., Luo, Y., Zhang, P., Li, Q. \& Xu, B. (2020). Primary tumor surgery for patients with de novo stage IV breast cancer can decrease local symptoms and improve quality of life. Annals of Surgical Oncology, 1-9. 10.1245/s10434-019-08092-2

Skaczkowski, G., White, V., Thompson, K. et al. (2018). Do Australian adolescents' and young adults' experiences of cancer care influence their quality of life? Psycho-Oncology, 27, 990-997. https://doi.org/10.1002/pon.4625

Songun, I., Putter, H., Kranenbarg, E. M. K., Sasako, M. \& van de Velde, C. J. (2010). Surgical treatment of gastric cancer: 15-year follow-up results of the randomised nationwide Dutch D1D2 trial. The lancet oncology, 11(5), 439-449. 10.1016/S1470-2045(10)70070-X

Souza, M. A., Freitas, R. W. J. F., Lima, L. S., Santos, M. A., Zanetti, M. L., \& Damasceno, M. M.C. (2019). Health-related quality of life of adolescents with type 1 diabetes mellitus. Rev. Latino-Am. Enfermagem, 27, e3210. 10.1590/1518-8345.2961.3210

Vlachioti, E. (2016). Assessment of quality of life of children and adolescents with cancer during their treatment. Jpn J Clin Oncol, 46(5), 453-461. https://doi.org/10.1093/jjco/hyw009

Wu Wei-Wen et al. (2015). The Mediating Role of Resilience on Quality of Life and Cancer Symptom Distress in Adolescent Patients With Cancer. Journal of Pediatric Oncology Nursing, 32(5), 304-313. 10.1177/1043454214563758

Ychou, M., Boige, V., Pignon, J. P. et al. (2011). Perioperative chemotherapy compared with surgery alone for resectable gastroesophageal adenocarcinoma: an FNCLCC and FFCD multicenter phase III trial. Journal of clinical oncology, 29(13), 1715-1721. 10.1.1.990.6934 